REPARATIVE CHANGES THAT TAKE PLACE ABOUT THE ROOT-ENDS OF INFECTED PULPLESS TEETH, AFTER TREATMENT

\title{
A Report of Cases From Practice
}

ARTHUR H. MERRITT

New York City

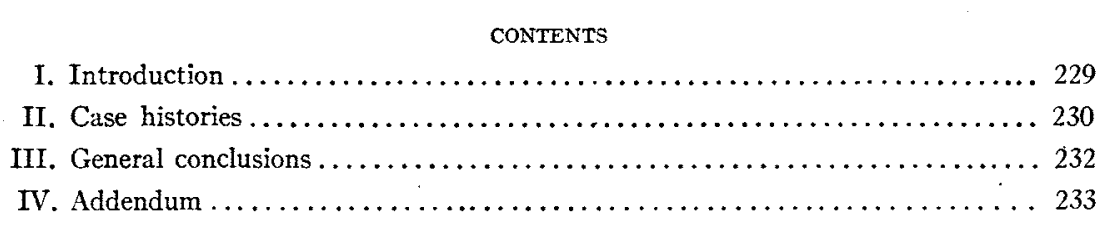

\section{INTRODUCTION}

That certain changes of a reparative nature take place about the root-ends of infected teeth as a result of treatment, by which they are apparently restored to health, as shown by comparative roentgenographic study, is no longer a subject for speculation. It is a fact susceptible of proof. What the exact nature of these changes is, what form of treatment is best adapted to produce them, and what conditions make for or against success, are questions which cannot be fully answered in the light of present knowledge. It is a fact, however, that under certain forms of treatment, including sterilization and root filling, many teeth showing marked periapical disturbance are apparently restored to health. Experience in clinical and roentgenographic examination has shown that when pulpless teeth are treated with sterilizing agents in such a way as to produce comparatively little injury to the periapical tissues, and when the root canals are filled to the end so as to seal them (all being done under aseptic conditions), excellent results are achieved.

The difficulties in the way of success would seem to be not so much in the form of treatment, as outlined above, as in complying 
with the conditions essential to success; and in being certain that they have been complied with in any given case. Where these conditions can be met with reasonable certainty (which is possible with most single rooted teeth, and, to a less extent with multi-rooted teeth) and where the case history is such as to justify conservative treatment, it is not unreasonable to believe that such teeth can be safely retained.

For the purpose of showing what may occur about teeth periapically infected, a few cases from practice, in which the prognosis was unfavorable, have been chosen to illustrate some of the reparative changes which take place, when the conditions above mentioned have been complied with. The case histories are given for the purpose of throwing as much light as possible on one of the most complex problems in dental practice.

\section{CASE HISTORIES}

Case 1. Age thirty-eight. Infected upper lateral incisor. Systemic symptoms suggestive of focal infection. Fig. 1 shows the tooth as roentgenographed on November 19, 1918. Fig. 2 shows changes which had taken place on November 4, 1919. It will be observed that complete regeneration has taken place in the periapical tissues. There was also disappearance of all systemic symptoms.

Case 2. Age seventeen. Cyst due to gangrenous tooth-pulp in lower right lateral incisor. History of occasional neuralgic pains on the right side of the face extending over a period of three years-the first molar had been extracted in an attempt to locate it. First seen January 25, 1919; fig. 3 shows a roentgenogram made on that date. Note the clearly defined outline of a cyst cavity. Subsequent to opening the tooth, acute symptoms developed with swelling of the face and discharge of pus through the tooth. These symptoms disappeared; after treatment, the tooth was filled on February 27, as shown in fig. 4. Fig. 5 shows results on June 14, 1920. All evidence of the existence of a cyst cavity had disappeared, and no further symptoms appeared.

Case 3. Age forty-two. Right upper first bicuspid with a so-called "blind abscess." Extraction was advised. Attempt at saving the tooth was urged by the patient. Fig. 6 shows a roentgenogram with a measuring wire in place on November 13,1916. Note complete loss of bone in the apical area. After treatment, the tooth was filled on December 11, as 
shown in fig. 7. Figs. 8,9 and 10 show progressive changes that took place up to June 10,1920 . The tooth has remained free from symptoms since treatment.

Case 4. Age thirty-nine. Lower left second bicuspid. Fistulous abscess, but no local or systemic symptoms. As the tooth was an abutment for a fixed bridge, treatment was urged by the patient. Fig. 11 shows a roentgenogram taken November 24, 1917. Observe the large abscess cavity and the eroded appearance of the root end. Fig. 12 shows the tooth as filled on January 28,1918 . The results of treatment are shown in fig. 13, taken January 5, 1921. Also note the improvement in the first bicuspid.

Case 5. Age forty-seven. Right upper lateral incisor. Blind abscess as shown in fig. 14, November 4, 1915. General symptoms, suggesting focal infection. No local symptoms. On opening the tooth, a watery discharge followed introduction of the instrument. Cultures made by the Research Laboratories of the New York Health Department showed a low grade staphylococcus. After treatment, the root was filled, November 30, 1915. All secondary symptoms disappeared. Figs. 15 and 16 show the processes of repair that had taken place, respectively, on February 1, 1917 and January 22, 1921.

Case 6. Age forty-four. Blind abscess involving central and lateral incisors, cuspid and first bicuspid, as shown in fig. 17, taken March 12, 1918. First bicuspid was extracted; the others were opened and treated. As the patient lived at a considerable distance, the roots of these teeth were not filled until November 13, 1918, as shown in fig. 18. Note the relation of the abscess cavity to all four teeth as shown in this roentgenogram. These teeth were crowned; four months later, March 28, 1919, another roentgenogram was made, as seen in fig. 19. It will be observed that repair in the apical area is already under way. Because of the distance at which this patient lives, later roentgenograms have not been made to date.

Case 7. Age twenty-seven. Pulpless lower second deciduous molar which had been retained owing to congenital abscence of second bicuspid. Fig. 20 shows a roentgenogram made December 2, 1919. Acute symptoms of pain, swelling and discharge of pus, followed opening of the tooth. After treatment, the roots were filled February 16, 1920. No symptoms appeared since filling. Fig. 21 shows the extent to which repair had progressed on January 13,1921 , less than one year after the case was dismissed. The unique feature of this case was the fact that the tooth under treatment was a deciduous molar.

IIIE JOUENAL OF DENTAL RESEARCH, VOI. III, NO. 1 
Case 8. Age thirty-six. Upper lateral as shown in fig. 22; roentgenogram taken May 10, 1919. Clearly defined apical area of radiolucency, with a perforation made in attempts to remove the root filling. Fig. 23 shows the result, January 22, 1921. This case is shown because of the complication caused by the perforation. The root was filled in the usual way, no attempt being made to fill the false canal. The tooth has been wholly free from symptoms since filling.

In all cases, tricresol and formalin, as advocated by Buckley, were used for sterilization. No other treatment was employed. Three to five treatments were made. The root canals were filled with chloro-percha and gutta-percha points. All were cases in which the prognosis was regarded as unfavorable and treatment was undertaken with some misgivings. For this reason these cases were kept under observation. In no case has any local or systemic symptom developed, since treatment, that would indicate continuance of infection in these teeth. These cases are reported here for the purpose of showing what may occur in periapically infected teeth, under initially adverse conditions, after treatment.

\section{GENERAT, CONCLUSIONS}

These results can undoubtedly be multiplied indefinitely and, in cases less marked, similar results are of almost daily occurrence, pointing clearly to the fact that, under certain conditions, pulpless teeth may be restored to health and usefulness. At least, these results indicate that no one may presume to be arbitrary in the matter. Indiscriminate extraction, as practised by some, would seem to be as unwise as indiscriminate attempts at saving all pulpless teeth. Sterilization by approved methods, and the filling of root canals to the end, appear to be the prerequisites to success in the treatment of pulpless teeth. Where these prerequisites can be met, and $_{\mathrm{i}}$ where there are no serious systemic complications indicative of focal infection, there is accumulating evidence that pulpless teeth can be safely retained. 


\section{ADDENDUM}

Since the foregoing was presented to this JouRNAL for publication, there appeared in this Journal an article by Novitzky, a reply by Rhein, and a rejoinder by Novitzky. ${ }^{1}$

Novitzky's statement, that "a careful examination of hundreds of devitalized teeth has failed to show a single one that, six months after devitalization, was not infected," means only that the conditions essential to success in the treatment of such teeth had not been met. Rhein is well within the truth when he says, in reply, "that a large percentage of teeth with non-vital pulps can be so treated as to leave them free to function, without any danger of such teeth becoming factors in focal infection."

The statement that "a pulpless tooth is in every sense of the word dead, for its only source of nourishment has been cut off," has been proved by Box to be incorrect. Those who saw his photomicrographic slides at the meeting of the National Dental Association in New Orleans in 1919 (copies of which have not yet been published) need no other proof of the inaccuracy of the statement that a pulpless tooth is necessarily a "dead" tooth. That there are pulpless teeth which cannot be saved goes without saying; but to insist that all such teeth are "dead" teeth, and should be extracted, is bad teaching and worse practice.

58 West 47th Sireet

${ }^{1}$ Novitzky: Journal of Dental Research, 1920 ii, p. 567; Rhein: Ibid., p. 579; Novitzky: Ibid., p. 585. 


\section{PLATES 1 and 2}

Figs. 1-13. Cases 1 to 4, inclusive. See histories, pages 230-231

Figs. 14-23. Cases 5 to 8, inclusive. See histories, pages 231-232 


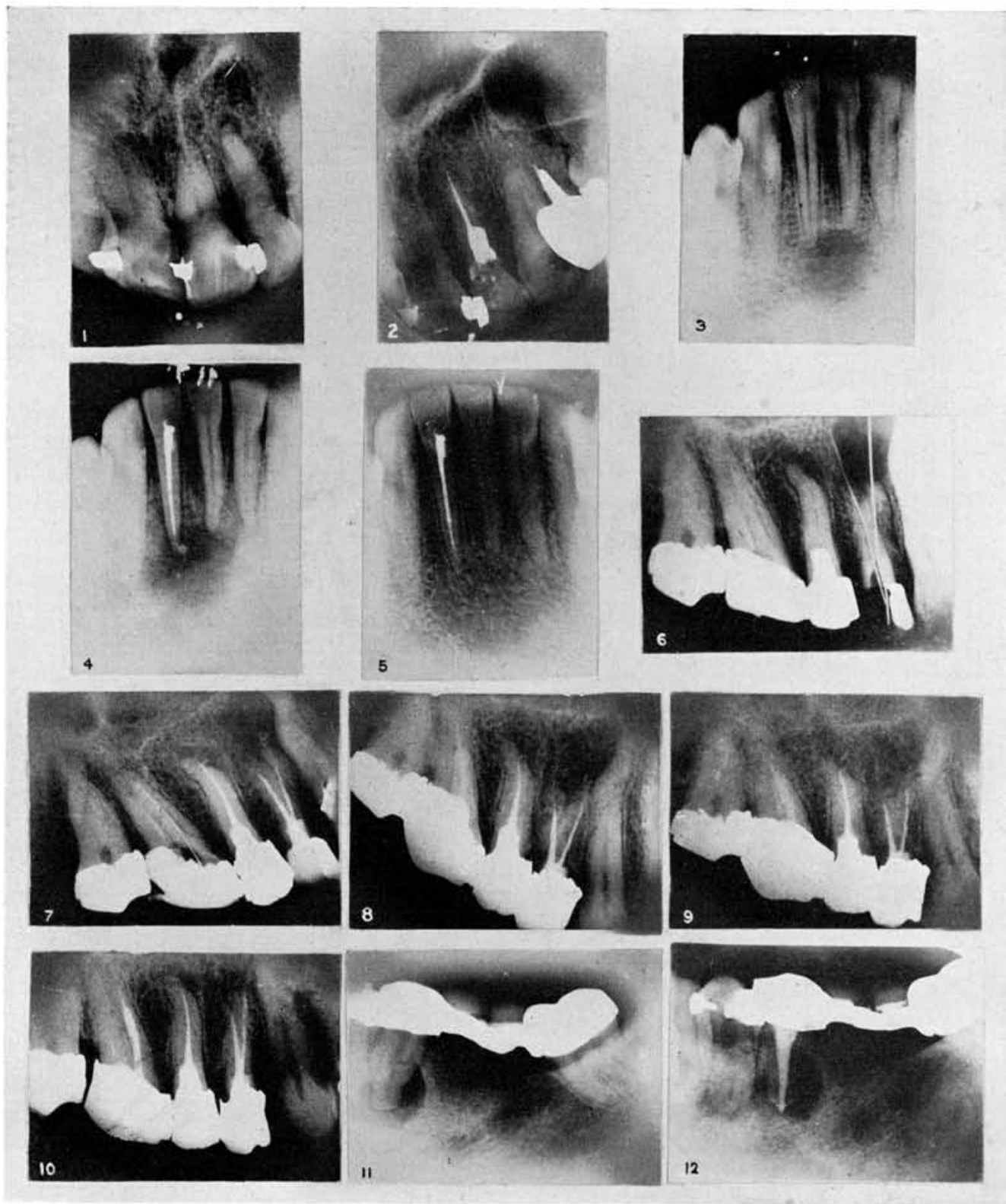

(Merritt: Periapical Repair After Treatment) 


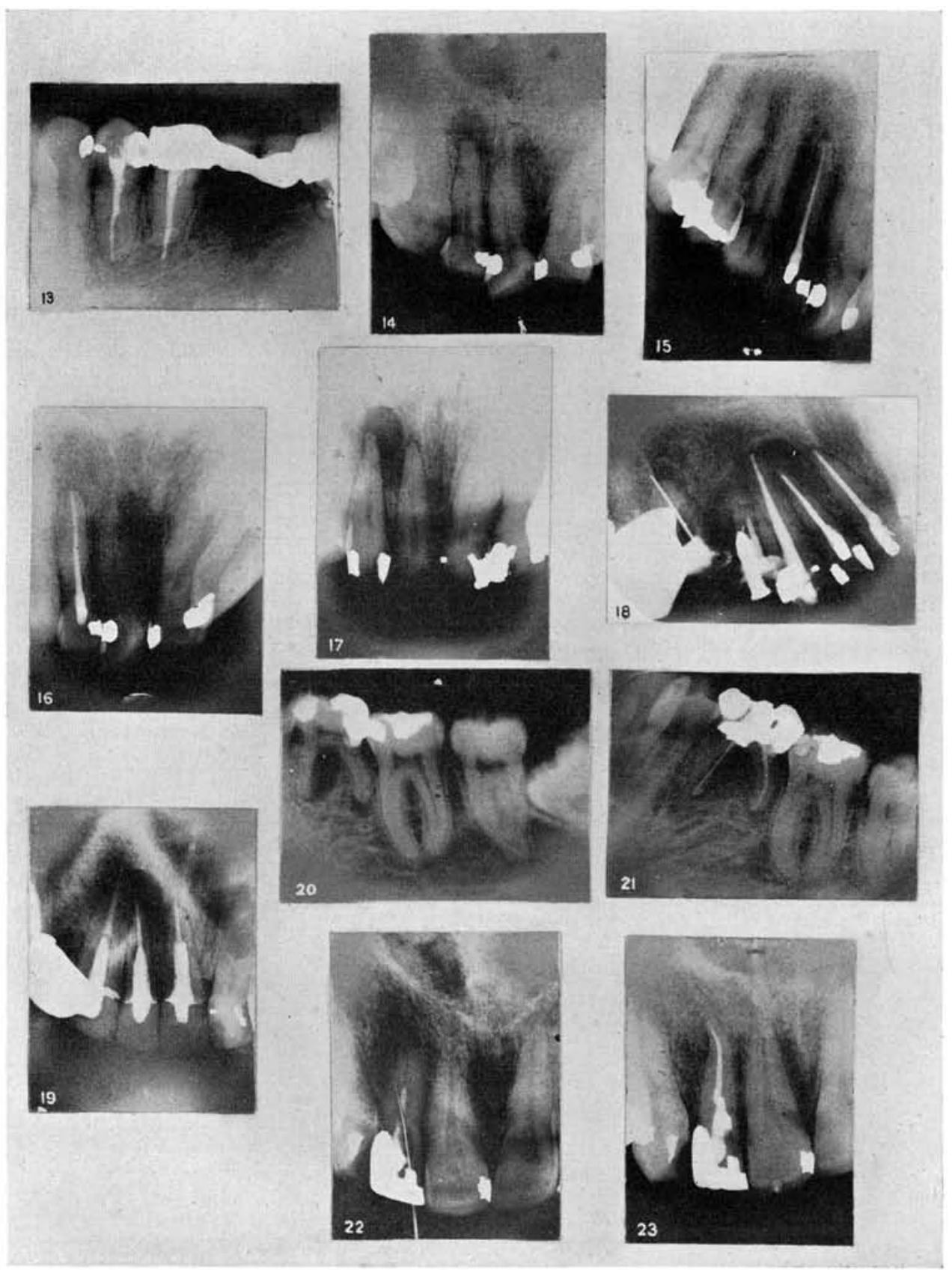

(Merritt : Periapical Repair After Treatment) 
